\title{
James G. Lochtefeld, God's Gateway. Identity and Meaning in a Hindu PilgrimagePlace
}

New York, Oxford University Press, 2010, 322 p.

Mathieu Claveyrolas

\section{OpenEdition}

\section{Journals}

Édition électronique

URL : http://journals.openedition.org/assr/26485

DOI : $10.4000 /$ assr. 26485

ISSN : $1777-5825$

Éditeur

Éditions de l'EHESS

Édition imprimée

Date de publication : 31 décembre 2014

Pagination : 239

ISBN : 978-2-7132-2467-6

ISSN : 0335-5985

\section{Référence électronique}

Mathieu Claveyrolas, « James G. Lochtefeld, God's Gateway. Identity and Meaning in a Hindu

PilgrimagePlace », Archives de sciences sociales des religions [En ligne], 168 | 2014, mis en ligne le 07 mai 2015, consulté le 22 septembre 2020. URL : http://journals.openedition.org/assr/26485 ; DOI : https://doi.org/10.4000/assr.26485

Ce document a été généré automatiquement le 22 septembre 2020

(C) Archives de sciences sociales des religions 


\section{James G. Lochtefeld, God's Gateway. Identity and Meaning in a Hindu PilgrimagePlace}

New York, Oxford University Press, 2010, 322 p.

Mathieu Claveyrolas

\section{RÉFÉRENCE}

James G. Lochtefeld, God's Gateway. Identity and Meaning in a Hindu PilgrimagePlace, New York, Oxford University Press, 2010, 322 p. 
$1 \quad$ Haridwar est l'une des sept villes saintes hindoues et l'un des principaux sites pèlerins de l'Inde du Nord. James Lochtefeld propose ici une étude monographique précise et bien documentée de cette ville de 220000 habitants connue pour abriter le «plus grand rassemblement religieux $\mathrm{du}$ monde ", la Kumbha Mela. Toujours soucieux de contextualiser ses données et analyses, l'auteur soulève un certain nombre de questions qui stimulent le lecteur, indianiste ou plus largement intéressé par les religions et les pèlerinages.

2 Lochtefeld donne pour titre à son ouvrage la traduction littérale de Haridwar: " porte de Dieu». Il explique en effet en détail comment la ville sainte est tout à la

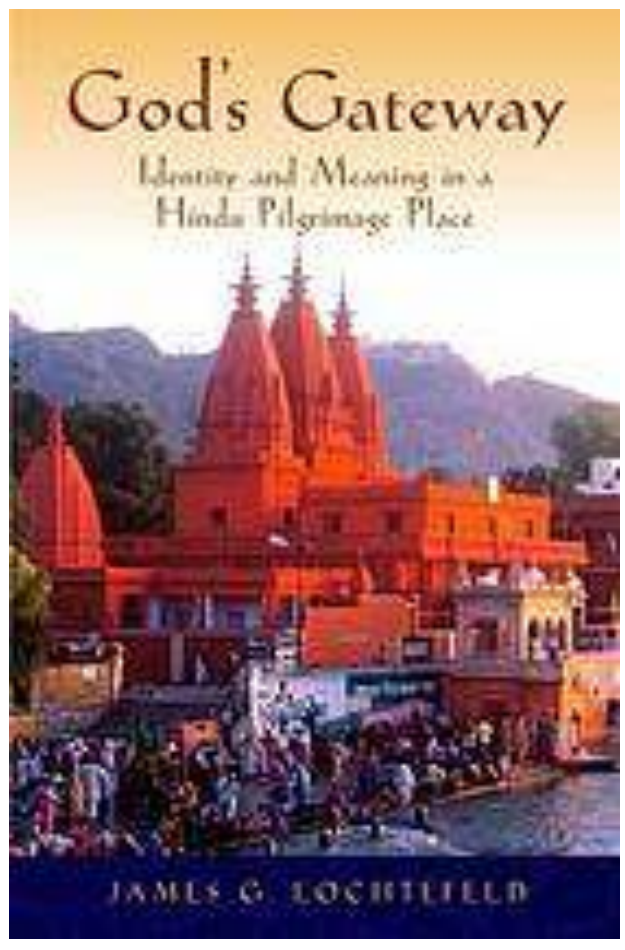
fois la «porte» physique pour accéder à l'Himalaya et à ses nombreux sites pèlerins hindous, et la " porte » symbolique vers le monde des dieux. Haridwar est bien un «tirtha », lieu saint hindou que l'on peut également traduire par « gué » ou « lieu de passage » où la proximité entre mondes divin et humain est maximale.

Le sous-titre (Identity and Meaning in a Hindu Pilgrimage Place) tente de rendre compte de l'approche anthropologique adoptée par l'auteur. L'ouvrage ne convainc pas définitivement de la pertinence de ces deux concepts tels qu'utilisés ici : ainsi définir le « sens » de la ville comme « l'attribution de certaines idées au lieu » (p. 8) semble trop général pour guider l'ethnographie ou stimuler l'analyse. On regrette parfois que l'auteur, qui vise également à une analyse du pèlerinage et de l'hindouisme en général (p. 103) limite souvent aux discussions méthodologiques la comparaison avec d'autres monographies de villes saintes hindoues, telles celles de Parry sur Bénarès (Jonathan Parry, 1994, Death in Banaras. Cambridge, Cambridge University Press) ou Van der Veer sur Ayodhya (Peter Van der Veer, 1988, Gods on Earth. The Management of Religious Experience and Identity in a North Indian Pilgrimage Centre. London, The Athlone Press).

4 James Lochtefeld a commencé son terrain à Haridwar dès 1989, et l'on sent une réelle familiarité avec la ville. Sans négliger les données historiques ou textuelles, l'auteur s'est personnellement investi dans son terrain, ainsi qu'en témoigne son récit très vivant d'un pèlerinage en bus (bus tour) dans l'Himalaya auquel il a participé. Il offre au lecteur un panorama à la fois ethnographique et contextualisé de la ville dans un équilibre très réussi. Soulignant à juste titre qu'il n'existe pas un seul et unique " récit de la ville» (p. 5), l'auteur insiste en outre sur la diversité des acteurs en jeu dans la construction de l'«identité » et du «sens » de la ville sainte, qu'ils soient pèlerins, prêtres ou ascètes notamment. Il s'attache également à témoigner des évolutions de Haridwar et du pèlerinage, en consacrant des chapitres à la mythologie, à l'histoire et aux changements contemporains. 
5 Outre l'écriture très agréable de l'ouvrage, le lecteur apprécie la clarté de son organisation en chapitres traitant successivement d'un type de discours sur Haridwar (les sources sanskrites, chapitre 2) ou d'un type d'acteur (les ascètes, chapitre 6).

6 Lochtefeld s'efforce de démystifier l'image idéale d'un pèlerinage hindou déconnecté des réalités et contraintes du quotidien. Il s'inscrit ainsi contre l'analyse de Turner et la pertinence du concept de communitas (p.172). Après tout, les pèlerins arrivés à Haridwar dorment (et mangent) traditionnellement dans la maison de leur prêtre héréditaire, qui les conserve dans un "entre-soi » (de caste, de langue et d'origine géographique) certain. L'auteur déconstruit également systématiquement, et à nouveau à juste titre, le «cliché [partagé par Indiens et Occidentaux] contrastant la spiritualité indienne avec le matérialisme occidental » (p. 160).

7 On sort ainsi du chapitre consacré aux ascètes convaincu que l'image occidentale du sage errant et détaché du monde est pour le moins partielle. Les ascètes se caractérisent au contraire par une violence souvent extrême, qu'elle soit tournée vers eux-mêmes dans le cadre de mortifications ou vers le monde alentour. L'image qu'en ont les Indiens est d'ailleurs, d'une manière générale, très ambigüe, mêlant la crainte et la méfiance au respect. Après tout, les pouvoirs magiques dont sont censés disposer les ascètes sont d'autant plus inquiétants qu'ils sont peu contraints par les normes et codes des non-ascètes. En outre, Lochtefeld explique parfaitement comment les ascètes, organisés en sectes (parfois martiales) rivales et loin de tout détachement envers les considérations mondaines, participent directement à la définition de la ville sainte comme un lieu de conflit sur fond de revendication statutaire. Dans ce contexte, le fait d'armes le plus spectaculaire, en 1760, aurait vu les ascètes sanyasis tuer 18000 de leurs rivaux bairagis!

De fait, l'approche historique de l'auteur démontre combien pareille vision démystifiée des ascètes ne peut être réduite à un éloignement récent d'un quelconque idéal de détachement qui aurait jadis prévalu. Le souci statutaire n'est d'ailleurs pas exclusif d'autres considérations, notamment économiques, tout aussi anciennes. Dès avant la période britannique, de puissantes sectes d'ascètes (notamment les naga sanyasis) contrôlaient efficacement le commerce de Haridwar, un privilège qu'elles ont su en partie conserver jusqu'à aujourd'hui, malgré les bouleversements apportés par le gouvernement britannique (notamment soucieux de contrôler les foules pèlerines et les conséquences sanitaires) puis par le gouvernement indien (qui règle désormais l'ordre des processions et des bains rituels).

9 De la même façon, la relation entre pèlerins et prêtres spécialisés dans le pèlerinage (pandas) est systématiquement discutée par Lochtefeld. L'auteur analyse la complémentarité entre prêtres et ascètes, et les multiples repositionnements statutaires conséquents. Il prend surtout soin de souligner l'évolution récente, contemporaine de l'émergence du tourisme et des classes moyennes, tendant à « réduire les pandas au statut de courtiers rituels» (p. 214) alors qu'ils étaient jadis les interlocuteurs héréditaires de familles de pèlerins pour qui ils servaient également d'hôtes et de guides. Parmi les autres transformations majeures de la vie pèlerine de Haridwar, on relèvera l'arrivée du train, en 1886. Lochtefeld souligne combien, malgré l'idéal voulant qu'on pèlerine à pied, le train a à la fois multiplié le nombre de pèlerins et bouleversé leurs comportements : on se rend désormais à Haridwar toute l'année (plutôt qu'au moment des foires et festivals), plus souvent (plutôt qu'une seule fois dans sa vie comme c'était la norme avant le train) et pour quelques jours seulement. 
L'évolution fut si vite intégrée aux mœurs, et l'emploi du train par les pèlerins si répandu, que, onze années seulement plus tard, les Britanniques purent tenter de prévenir une épidémie de peste en arrêtant la circulation du train pour Haridwar, limitant ainsi radicalement l'ampleur des rassemblements. Effectivement convaincus que cela causerait la désaffection des pèlerins, et la chute de leurs revenus, les pandas se plaignirent amèrement aux autorités britanniques (p. 71).

On aura compris que l'auteur s'inscrit dans la lignée de Eade \& Sallnow, notamment via la description d'une ville de pèlerinage comme « arène de discours séculiers et religieux conflictuels ». Tout en souscrivant à cette approche et à l'excellente illustration qu'en fournit l'ouvrage, on regrettera un certain déséquilibre dans le traitement des données, qui dessert parfois la profondeur de l'analyse. Ainsi le vocabulaire utilisé, visant sans doute à démystifier certains clichés, semble-t-il parfois trop systématiquement réducteur. Pour ne citer que quelques exemples, les textes sacrés sont des " publicités " (advertisement, p. 6, p. 46), le pèlerinage un «carnaval» (carnival, p. 15, p. 108), les ascètes comptent de nombreux « charlatans », « sans scrupule » et « égoïstes » (p. 157), les pèlerins sont des «clients" (customers, p. 171) dont la mentalité est celle de "vacanciers» et l'éthos celui du «consommateur» (vacation mentality, p. 204 et consumer ethos, p. 211).

11 Pareil vocabulaire réduit parfois trop vite l'analyse à la déconstruction et ne laisse pas suffisamment de place à la discussion de tensions qui sont pourtant cruciales pour notre appréhension de l'hindouisme, telles que celles entre spiritualité et pragmatisme, entre dévotion et rite ou entre politique et religion. Ainsi lorsque l'auteur s'étonne qu'un « lieu sacré » (holy place) ne "garantisse pourtant pas une atmosphère religieuse saine et pure [pure and wholesome religious atmosphere]» (p. 108), on en reste à une remarque ethnocentrée en l'absence d'une discussion des conditions hindoues de l'expérience religieuse et des conceptions hindoues du sacré. Autre exemple : la notion d'honoraire sacrificiel (daksina) est tout juste évoquée (p. 131) et les arcanes hindous du don (dan) à peine davantage (p. 132), rendant trop partiale l'analyse des dimensions purement financières de la négociation entre pèlerin et panda. À notre sens, l'approche est plus justement équilibrée lorsque l'auteur, concluant sa discussion sur la politisation récente du pèlerinage hindou, écrit : «il serait pourtant absurde [silly] de prétendre que les éléments nationalistes hindous contrôlent à distance» (p. 220) les processions. En effet, les principales évolutions du pèlerinage hindou (influence du tourisme ou de la politique) parfaitement illustrées par l'ouvrage ne doivent pas cacher la stabilité du phénomène dans le temps ni l'importance pérenne première de sa dimension d'expérience religieuse.

En conclusion, s'il est certain que le lecteur peu rompu aux complexités de l'hindouisme sera utilement édifié par la présentation démystifiante de l'auteur, le lecteur indianiste sera lui parfois frustré par l'absence de pistes d'analyses véritablement nouvelles. Ces critiques ne doivent pourtant pas mener à négliger cet ouvrage qui, de par son projet monographique nourri de matériaux solides et très clairement présentés, constitue un apport non négligeable à la bibliographie traitant des villes sacrées et pèlerinages hindous. 